\title{
Seeing Blocks and Crypto Bros
}

\author{
LIAT BERDUGO \\ Assistant Professor, University of San Francisco \\ EMILY MARTINEZ \\ Independent Artist
}

\begin{abstract}
How do cryptocurrencies like Bitcoin work? What is a blockchain? And, what does a typical Bitcoin holder look like? The artist collective Anxious to Make commissioned sixty cloudworkers to draw their answers to these questions. Drawings range from the mundane and stereotypical-a Bitcoin owner depicted as a bearded hipster shouting, HODL! - to the complex and eccentricthe blockchain drawn as connected nodes of random numbers, presumably meant to represent cryptographic hashes.
\end{abstract}

Like much technology, the inner workings of cryptocurrency remain "black boxes" of understanding to most users. As a collective, Anxious to Make remains cautious of any technological apparatus that is believed in without understanding. These series of drawings point to the absurdity and comic nature of technological beliefs and utopic, seamless crypto-fantasies. We offer a subset of the drawings here, together with an analysis of the ways artistic labor is changed by — or mirrors - the practice of outsourcing.

\section{INTRODUCTION}

Inside the bowels of the third industrial revolution, where industry 4.0 meets crowdsourcing, where digital requests meet physical labor, lies the so-called "micro-task": a discrete unit of work that has been farmed out to diffusely located human laborers by means of a technology platform. TaskRabbiters assemble other people's Ikea furniture, Fiverr contractors record voice-overs for other people's commercials, and Mechanical Turk workers click away at other people's tasks on their computers. Laborers who are technically their own bosses are paid small sums by large corporations on a per-"micro-task" or "micro-job" basis, in what has become a burgeoning and fragmentalized economy of "micro-work." Meanwhile the income gap widens between the entrepreneur, the user, and the lowly serf who executes jobs under the phraseology of the extremely small (the "micro"). In this economy can a person ever really be their own boss, or merely their own "micro-boss"? 
Since 2015, we - the art collective Anxious to Make-have focused our work on economic concepts entailed in digital labor, which sociologist Antonio Casilli describes as "a continuum of unpaid, micropaid, and poorly paid 'taskified' human activities." $\mathrm{We}$ employ absurdist extremes to examine contemporary economic realities. One of our video pieces stars hired spokespeople from the gig-platform Fiverr.com, whom we paid to "quit" these temporary jobs of acting as spokespeople on our video. ${ }^{2}$ It was an act no stranger than if an Uber driver were to pull up to the curb and enthusiastically thank their rider for hiring him. Of course we all know the algorithm did it; but the driver is technically the boss and the rider is their summoner. Drivers pay their own taxes and maintain their own car insurance. As economists of the digital labor admit, we don't openly force or manipulate workers today; instead, we allow economic factors to invisibilize the economic forces of labor.

In tracing invisible labor through economies, we have often found ourselves on the landing page of Amazon's Mechanical Turk. Mechanical Turk, or "MTurk" for short, employs contractors to perform a micro-tasks called "Human Intelligence Tasks" (HITs) that require large amounts of data entry and are currently more efficiently or accurately performed by human beings than by computers. Mechanical Turk workers, or "MTurkers," complete tasks for as little as $\$ 0.01^{3}$ and earn a miserly median hourly rate of $\$ 1.38 .^{4}$ At the same time we have grown increasingly intrigued (and horrified) by the digital economy of cryptocurrencies like Bitcoin and the consolidation of extreme wealth and colonialist endeavors they have created in the name of decentralization. $^{5}$

Amazon's Mechanical Turk does indeed have, surprisingly, a good deal in common with the concepts that undergird cryptocurrency. First, both operate through networks in which no individual node comprises the full picture: there is no single node of the blockchain that holds the authority to act individually, nor is an MTurk worker generally hired to complete a HIT on their own. Technically, a requester could choose to request a HIT that accepted only single response, but this is generally not how the MTurk platform is used, as answers are formed from a conglomoration of completed micro-tasks.

Second, both MTurk and cryptocurrencies work by shifting trust away from experts and institutions. Satoshi Nakamoto famously wrote that the "root problem with conventional currency is all the trust that's required to make it work," notably, trust in central banking institutions staffed by trained economists, and the governments who back them. ${ }^{6}$ Cryptocurrencies still require trust, but that trust has shifted from a Leviathan trust in institutions to a distributed trust in a technology. Users on the blockchain trust the algorithms and protocols of the cryptocurrency rather than trusting an institution. Likewise, a requester who turns to MTurk with a task is willing to trust not in an expert, but in the collective data amalgamated from hundreds of responses. This trust emerges - almost phenomenologically - from hundreds of submitted responses to HITs, and it is not trust in any single particular MTurker. It is true that Amazon has created a class of "high 
performance" workers upon whom it bestows the expert status of "Mechanical Turk Masters" based on statistical monitering of completed HITs in order to economize on "expert" status.7 However, while some worker forums report that a "Masters" qualification is bestowed only after a worker completes 1,000 HITs with at least a $99.0 \%$ approval rating and continued activity, evidence suggests that the requirements are far more random. ${ }^{9}$ Therefore, the algorithmically determined expertise of workers stands as a mere bonus for which Amazon can charge extra money. MTurk remains a platform for requesters who trust not in the single expert, but in the mass of cheap labor that coheres into a trustworthy dataset of collective responses.

Finally, both Mechanical Turk and cryptocurrencies are technological platforms that are generally not well understood. Mechanical Turk's "Getting Started" guide clocks in at a whopping 17 pages long, ${ }^{10}$ and it has taken a three-person team of scientists armed with advanced probability functions to even calculate how many workers the platform employs. ${ }^{11}$ Likewise very few understand the mathematical underpinnings of cryptocurrency nor its exact decentralized structure - an issue which many on the internet attempt to remedy with a plethora of explainer videos and articles. Our personal favorite is the satirical essay by artist Cara Rose Defabio titled "Men Explain Blockchain to Me," which interjects a feminist critique into the oft-mansplained domain of crypto. ${ }^{12}$

Seeing these similarities between Mechanical Turk and cryptocurrency, we wondered what it might be like to launch a project that enmeshed the two. Frankly, we were curious what MTurkers would think about crypto. We created three HITs on MTurk with the prompts, "Draw how crypto works," "Draw the blockchain," and "Draw a person who owns Bitcoin," and specified that no advanced knowledge of cryptocurrency was required. We paid $\$ 0.25$ per drawing and accepted all responses provided that they were in fact drawings. We collected the results into a book titled "Seeing Blocks and Crypto-Bros" (2018).$^{13}$ Together, the drawings are fascinating in what art critic Blythe Sheldon called "their varying levels of detail, sloppiness, humor and confusion." We offer a subset of the drawings here, together with a few words of analysis, and finish with a consideration of the ways artistic labor is changed by—or mirrors - the practice of outsourcing. 


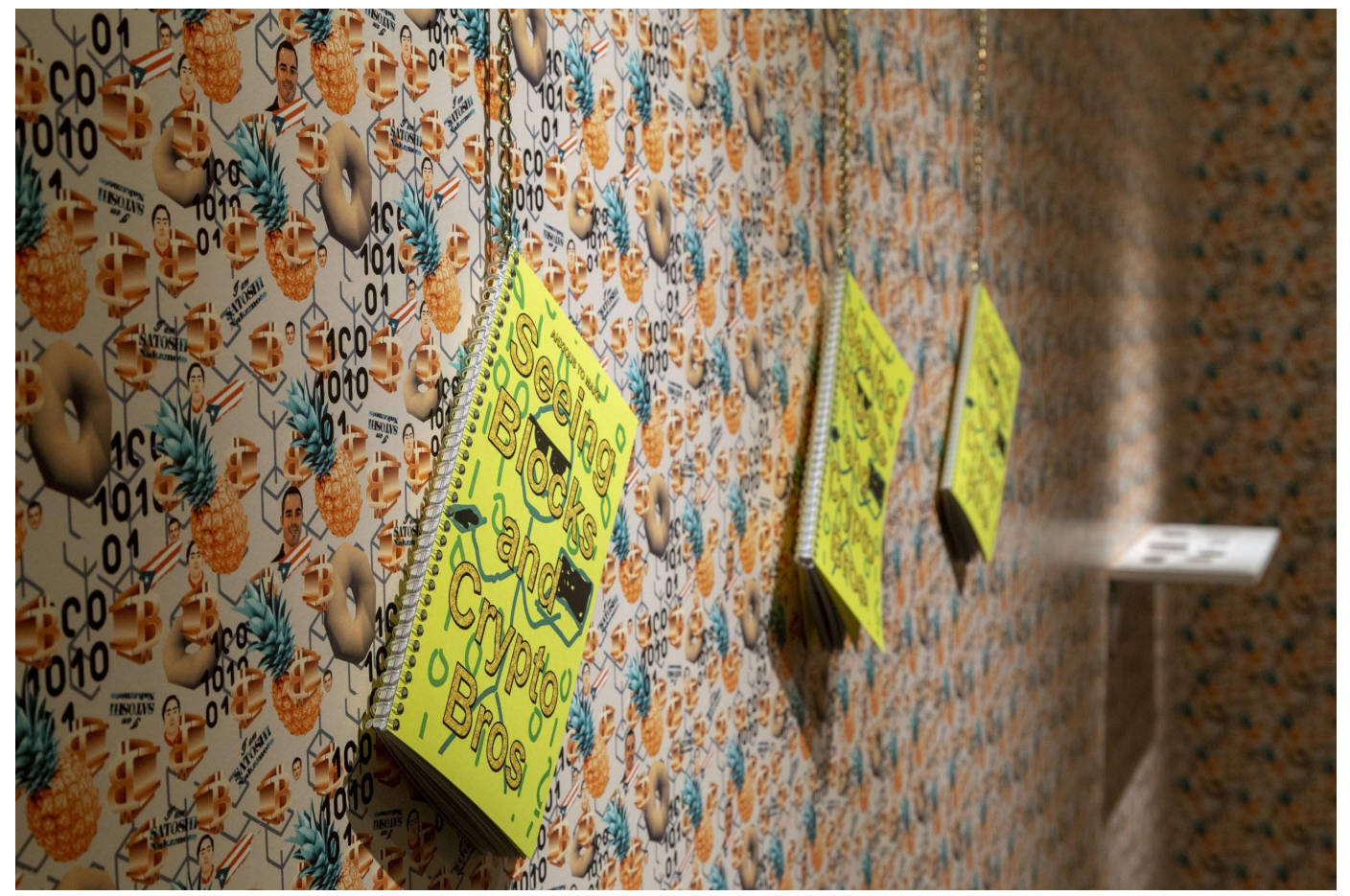

Figure 1. Seeing Blocks and Crypto Bros books on display as part of Eternal Boy Playground, Anxious to Make's solo exhibition at Telematic (San Francisco, CA) 2018, (C) 2018, Anxious to Make.

\section{"DRAW HOW CRYPTO WORKS"}

We asked Mechanical Turkers to draw how cryptocurrency works in their own understanding. The drawings, which were completed in the online drawing platform AutoDraw, ranged widely in content and drawing style. Many drawings centered on a computer as a visual depiction of the digital, as in Figure 2: this worker drew money, depicted as US Dollars, being transformed into a pile of gold coins on a computer screen via a magical red arrow. The computer is accompanied by a keyboard, drawn with just enough lines to understand the representation, and is situated on a brown desk in perspective. Other drawings focused on the peer-to-peer quality of cryptocurrency, as in Figure 3. This MTurker drew an exchange of money between two stick figures in blue, the default color of the drawing program. To drive their point home the MTurker drew a bank and crossed it out with a bold, red "X," changing color for the first time to highlight the noninvolvement of financial institutions. 


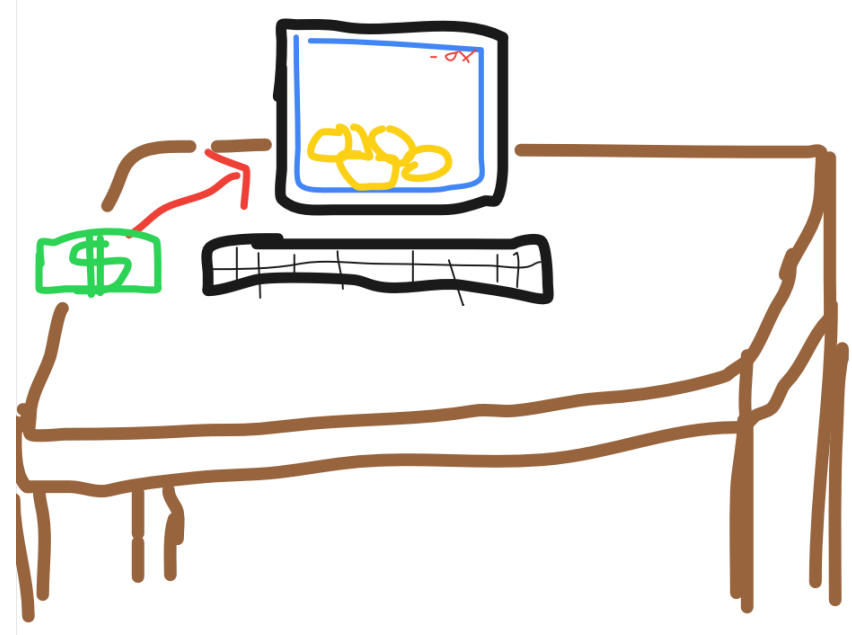

Figure 2. Draw How Crypto Works, Worker ID A22I5RTLJTY3JZ, Completion time 29m 29s, (C) 2018, Anxious to Make.
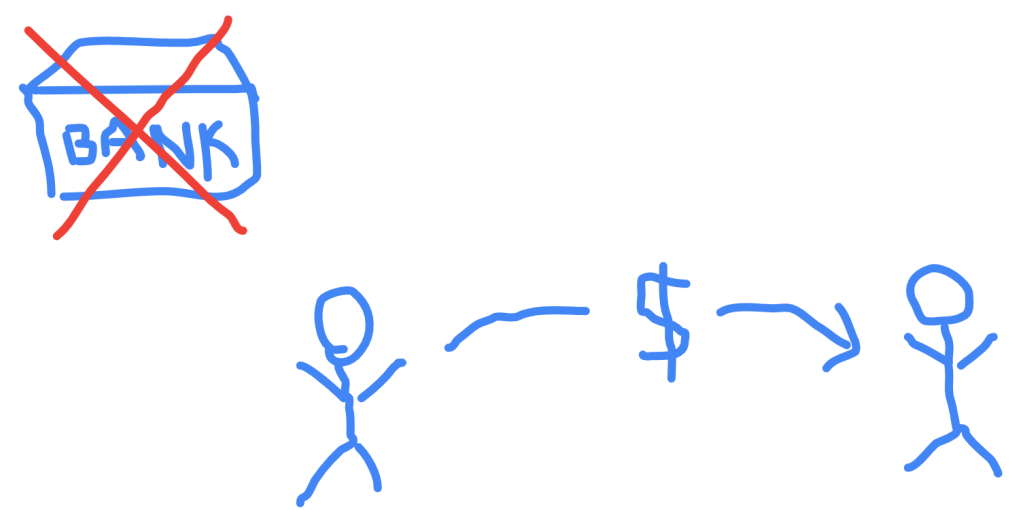

Figure 3. Draw How Crypto Works, Worker ID A2KYOM2ZGYDJGT, Completion time $9 m$ 39s, () 2018, Anxious to Make.

Other drawings chose to portray a larger view of the cryptocurrency system, involving not merely people or their personal computers but also server farms. In Figure 4, an MTurker drew the Bitcoin icon largely as the center of a network whose ties reached out to a laptop, a desktop, and a set of six servers. We are to understand that the servers are "mining" Bitcoin by the inclusion of a rock mining pick, the kind of tool used to chip away at mountain ore deposits (with the sound, "ting ting ting") in what we understand as the original definition of "mining." As the server farm 
mines Bitcoin it radiates heat, so the MTurker has drawn a fan to cool it down. Indeed the Bitcoin logo itself appears to be giving off heat, or perhaps it is a radiant glow of wealth.

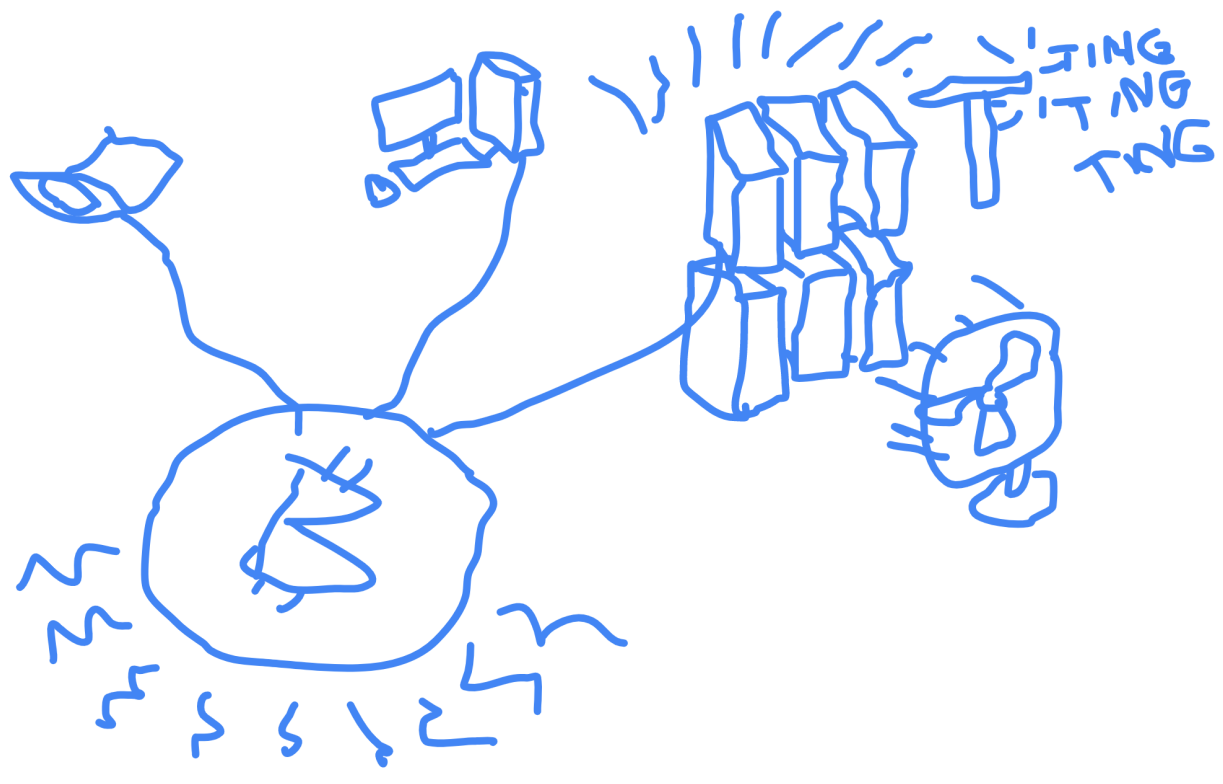

Figure 4. Draw How Crypto Works, Worker ID A2KNDTYE4PUIQ1, Completion time 8m 20s, (C) 2018, Anxious to Make.

Other drawings depict a networked picture of consumerism enabled by cryptocurrency. In Figure 5 , for instance, US dollars are converted into Bitcoin, which then appears to be used on a website to anonymously purchase some kind of unidentifiable product. The product is delivered to the happy shopper's front doon on a truck. The shopper is not depicted greeting the delivery, but picking it up from the doorstep of their home later. The drawing rings with overtones of Silk Road, the darknet marketplace which was shut down in 2013 for selling illegal substances and contraband.

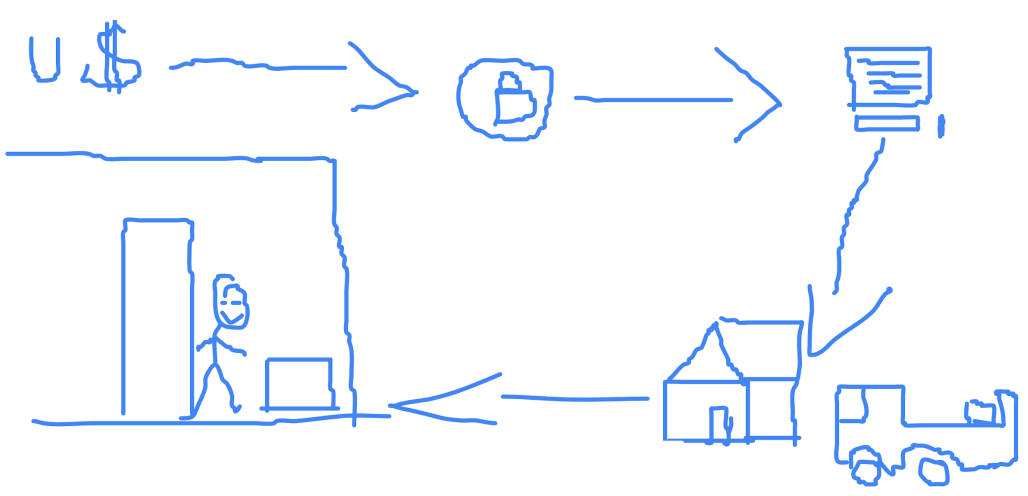


Figure 5. Draw How Crypto Works, Worker ID: A2D342FWN7IG35, Completion time 6m 09s, (C) 2018, Anxious to Make.

\section{"DRAW THE BLOCKCHAIN"}

The second task we commissioned on Mechanical Turk was a request to draw the blockchainthe technology that undergirds cryptocurrency - or anything that MTurkers thought resembles it. While it is common to conflate Bitcoin (the currency) with the blockchain, we found that MTurkers overwhelmingly drew networks, either as strings of connected nodes that generated infinite spirals of results (Figure 6) or as a more commonly portrayed web of nodes, each linking to many other nodes in what becomes a dense network of connections (Figure 7). This drawing in particular resembles popular representations of the World Wide Web.

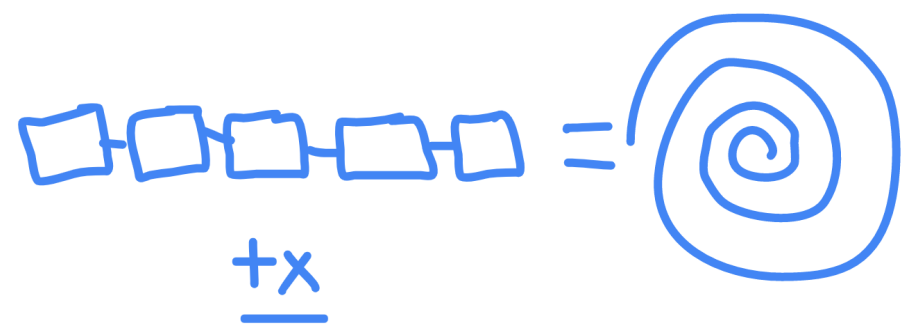

Figure 6. Draw the Blockchain, Worker ID A2B4GWF7MF7AOB, Completion time 38m 32s, (C) 2018, Anxious to Make.

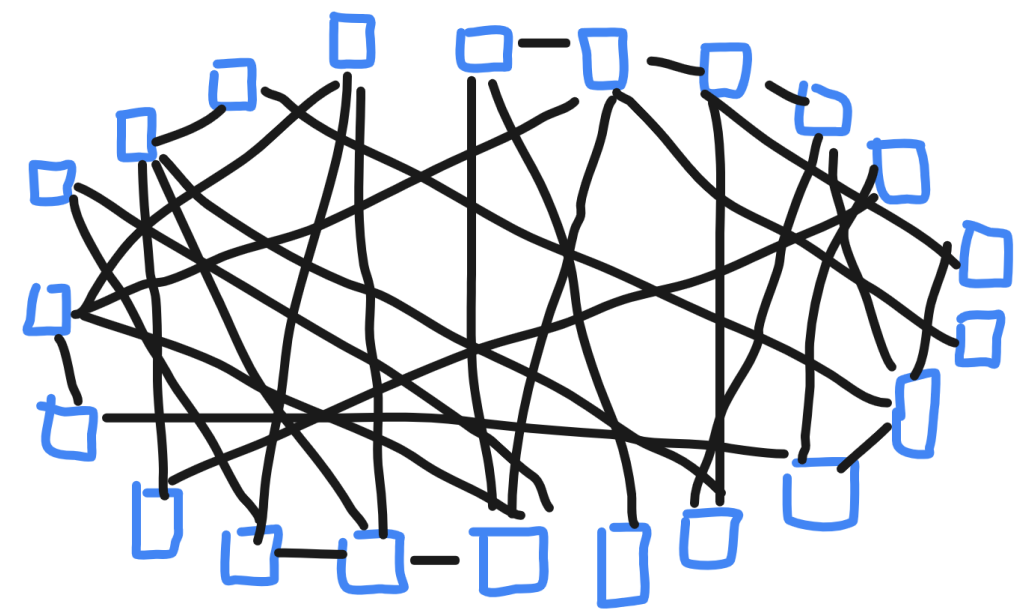

Figure 7. Draw the Blockchain, Worker ID AENW881YO2H8S, Completion time 3m 10s, (C) 2018, Anxious to Make. 
Others chose to highlight the complex mathematics that enable the blockchain's security. For instance Figure 8 shows two Bitcoin users exchanging currency, and their transaction is entered into a serial link of blockchain transactions that symbolizes the ledger. The drawing includes mathematical expressions (" $\sqrt{4}$ " and " 23 ") and functions (" $8 \mathrm{x}+\mathrm{N}=\mathrm{Y}$ "), which gesture toward the cryptographic hash functions that encrypt transactions on the public ledger. Never mind that the square root of four is easy to compute: the drawing's intention is to mobilize non-quotidien mathematical notations to indicate complexity.

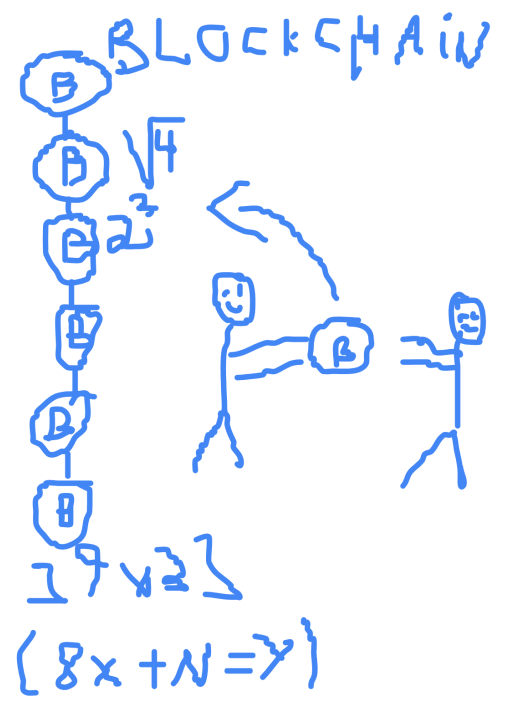

Figure 8. Draw the Blockchain, Worker ID A2D342FWN7IG35, Completion time 9m 29s, () 2018, Anxious to Make,

One of our favorite drawings of the blockchain represents it as a literal chain (Figure 9). The chain links a desktop computer with what appears to be a giant ornamental Bitcoin —or perhaps a blingy Bitcoin necklace - which is encircled by three loving hearts.

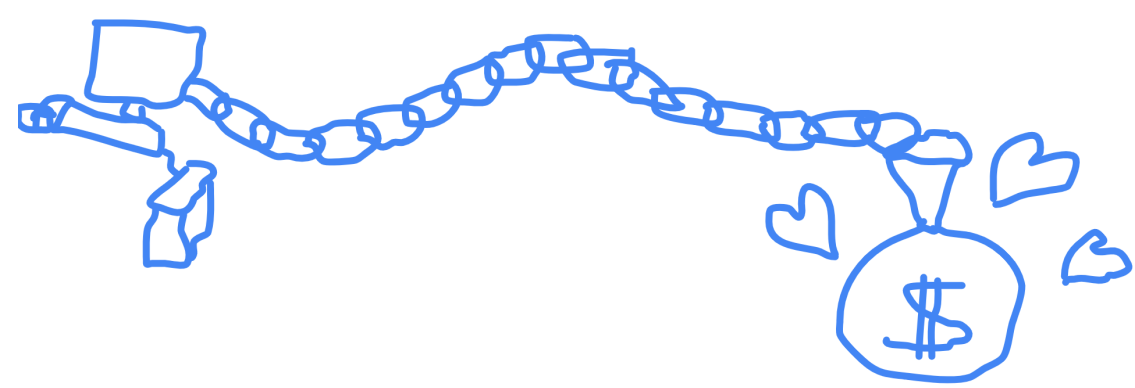




\section{"DRAW A PERSON WHO OWNS BITCOIN"}

The final prompt we commissioned on Mechanical Turk was to draw a typical person who owns Bitcoin, in the MTurker's own understanding of what that means. Perhaps unsurprisingly most of the Bitcoin owners were depicted as men, and often as persons who celebrated their status as wealthy, cool, or technologically adept. One drawing shows a man holding a laptop in one hand and a cellphone in the other as bytes of data rain down behind him, reminiscent of scenes in The Matrix (Figure 10). The man's sunglasses are his only accessory, and give off a relaxed, hip sense of pride at the technological devices in his possession. Another drawing depicts a Western-style businessman clad in a dress shirt and tie and smiling to reveal a gold tooth or "grill" in the back of his mouth (Figure 11). His tooth betrays his wealth, as does the car behind him.

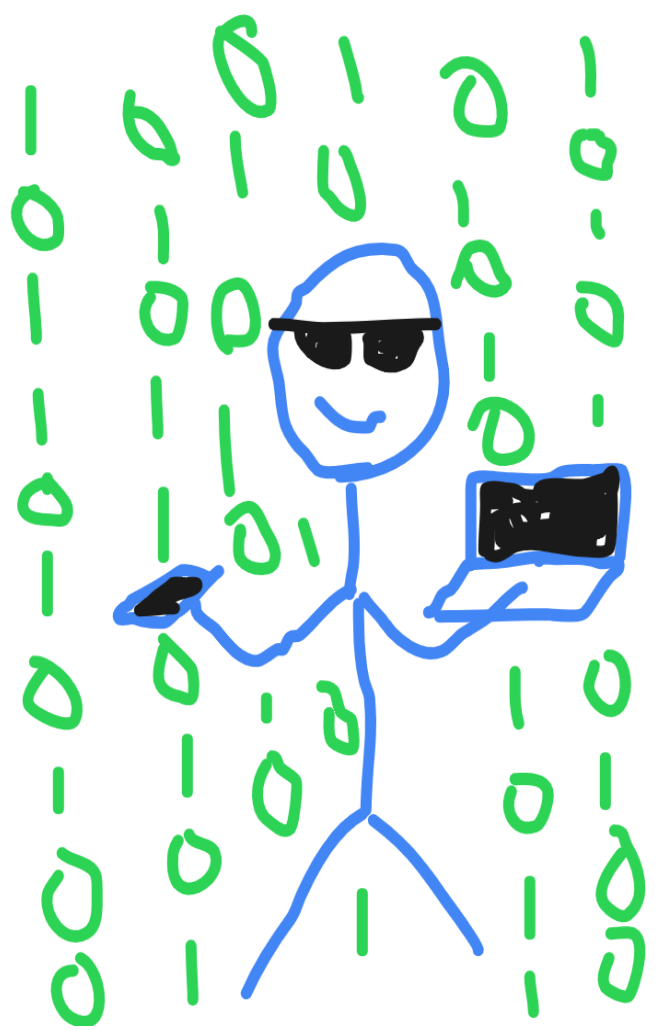

Figure 10. Draw a Person Who Own Bitcoin, Worker ID A2KYOM2ZGYDJGT, Completion time 13m 34s, @ 2018, Anxious to Make. 


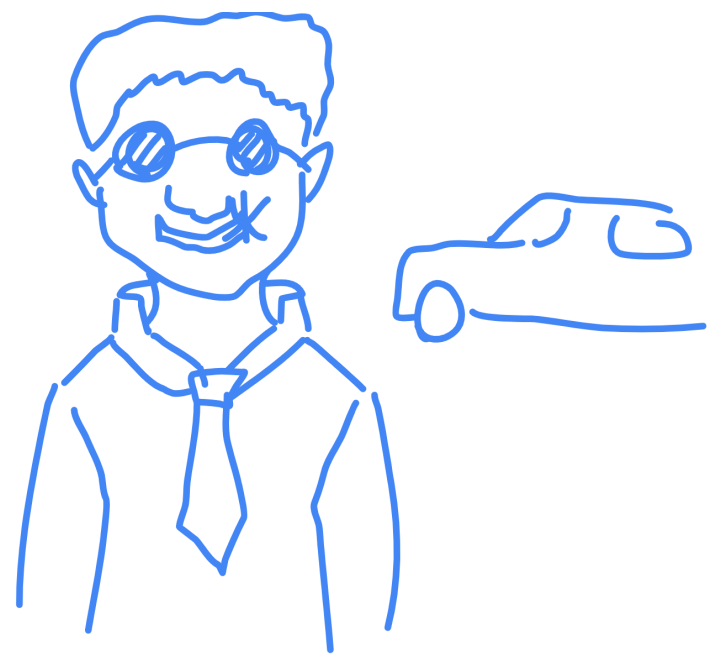

Figure 11. Draw a Person Who Own Bitcoin, Worker ID A157L6Z8QU3SDE, Completion time $29 m$ 57s, () 2018, Anxious to Make.

Another drawing portrays a Bitcoin owner as a nerd wearing a MAGA (Make America Great Again) hat, an iconic symbol of President Donald Trump and of right-wing politics more broadly (Figure 12). Digital studies scholar David Golumbia has written about the connections between cryptocurrency and far-right politics, arguing that Bitcoin supporters espouse a kind of cyberlibertarianism that relies heavily on extreme-right political thought. ${ }^{15}$ It is as if this drawing of a Bitcoin owner in a MAGA hat were a perfect visual summary of Golumbia's research.

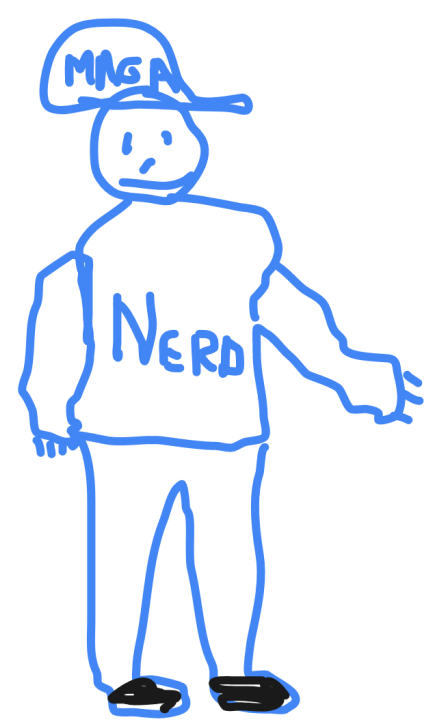

Figure 12. Draw a Person Who Own Bitcoin, Worker ID A2SQNS3J389FRJ, Completion time $3 m$ 15s, (C) 2018, Anxious to Make. 
Finally, MTurkers also depicted Bitcoin owners as miserable in the fall of the currency's worth. Figure 13 shows an unhappy stick figure viewing the Bitcoin price index graph on his computer. The price has spiked but has now steadily decreased. But the Bitcoin owner is not cashing out on his crypto-investments. Instead he is saying "HODL," an internet slang term derived from the misspelling of "hold." HODL refers to the buy-and-hold, never-sell strategy of Bitcoin and crypto-enthusiasts and betrays a deep and optimistic faith in the rising value of cryptocurrency, even in financial slumps. The drawing captures the notion that owning Bitcoin is often about much more than money: it is about identity, ideals, and even politics.

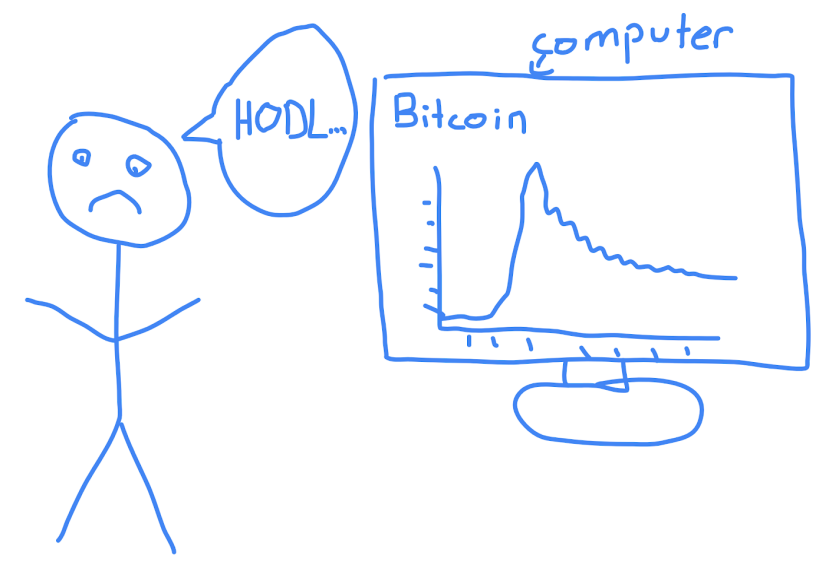

\section{Figure 13. Draw a Person Who Own Bitcoin, Worker ID AQVQHML14YYJH, Completion time 4m 07s, @ 2018, Anxious to Make.}

\section{ON OUTSOURCING ARTISTIC LABOR}

Our project is certainly not the first to outsource labor from Amazon's Mechanical Turk. Before us come drawing projects like Aaron Koblin's The Sheep Market (2006), a collection of 10,000 sheep drawings made by MTurkers who were each paid $\$ 0.02$ to "draw a sheep facing to the left," and which Koblin later sold as limited edition blocks of lickable adhesive stamps for $\$ 20$ each. ${ }^{16}$ Artists like Clement Valla have used Mechanical Turk to celebrate the imperfect humanity of MTurkers in his project, A Sequence of Lines Traced by Five Hundred Individuals (2011). ${ }^{17}$ Valla asked a series of MTurkers to trace a straight line, and each worker was presented with the most recent line drawn and thus traced the most recent imperfect copy. The line becomes wavy, bent and ultimately fragments into 18 squiggles. Lauren McCarthy, an artist who has long been open about her social anxiety, used real-time feedback from MTurkers to help her improve her social interactions in Social Turkers (2013). ${ }^{18}$ Instead of celebrating the diversity of MTurkers' responses, or celebrating their humanity in the face of mechanization, McCarthy positioned herself as "less than" the MTurkers and in need of their advice. 
Outsourcing artistic labor is certainly not new, nor is it limited to Mechanical Turk. Popular artists such as Jeff Koons and Damien Hirst openly employ throngs of assistants to produce their work, and indeed Koons brags that he never touches a paintbrush. ${ }^{19}$ Andy Warhol called his art studio a "factory" as an act of deskilling artistic labor, instead foregrounding the mechanical labor of the production of his silkscreens; it is a matter for art historical debate as to whether his prints were pulled by Warhol's hands or by his assistant's. Likewise, Sol LeWitt's wall drawings are not drawn by LeWitt himself but are instead distributed as sets of mathematical instructions for gallery workers to instantiate. Indeed, the through-line of artistic labor outsourcing can even be traced back to the Fluxus movement in the 1960s, in which artists wrote sets of whimsical instructions that they never performed, which they delegated to the audience to perform, or which indeed were never materialized at all.

More recently artists such as Alexander Tarrant have openly embraced the delegational or managerial role of the artist and have left the completion of the artwork to others. For Outsource Show: Paintings by Non-Painter Artists (2016), Tarrant invited a group of non-visual artists (such as writers, musicians, and filmmakers) to conceptualize ideas for paintings that were then painted by workers in China's Dafen Oil Painting Village. ${ }^{20}$ Michael Mandiberg's Postmodern Times (2018) is a fully outsourced movie: Mandiberg commissioned freelance workers on the platform Fiverr.com to recreate scenes from Charlie Chaplin's film Modern Times as an act of reimagining what industrialization means in the current era. ${ }^{21}$ Artists have even outsourced the labor of creating work to non-human living species. Polish artist Agnieszka Kurant, for instance, used millions of termites to create brightly colored sculptural mounds for a work titled Artificial Artificial Intelligence (2014). ${ }^{22}$ Art historian and critic Claire Bishop might call the termites' act a "delegated performance" of sorts-namely, an artistic performance act undertaken on an artist's behalf. $^{23}$

Many of these works of art - ours included - might be subjected to the derogatory phrase commonly uttered by viewers upon seeing modern or contemporary art: "My kid could do that."24 In Work Ethic, Helen Molesworth notes that this statement expresses dismay that artworks appear to be made without skill or labor. Indeed a related question asked of artworks, "How long did it take to make this?" explicitly questions artistic labor by seeking to quantify it in units of time with the idea that art should require "man-hours." But Molesworth notes that after World War II, the US economy shifted from a manufacturing-based economy to a service-based one, altering the labor conditions for a vast number of workers. Like the rest of the economy, art changed at this time, too, and moved away from the production of handmade, skilled, and labor-intensive objects. Instead, Molesworth explains, art began to be made "with unskilled manual labor, with highly regimented managerial labor, or with labor that resonated with ideas borrowed from the service economy." 25 Art made in these modalities did not look like art, argues Molesworth, much as sitting at a desk did not look like working on an assembly line. 
Work has undergone yet another transformation in the age of the so-called "sharing economy" or gig economy. Workers no longer sit at desks; instead they ferry around passengers in their personal cars for Uber or Lyft, rent their spare rooms out to temporary guests on AirBnB, and execute digital tasks on Fiverr or Mechanical Turk. Work happens anytime, day or night. Workers are independent contractors and as such no no longer work for anyone in particular. Likewise, art has followed in work's transformation and has explored modalities of production that include the continued outsourcing and fractalization of labor into an era of micro-work.

One of our first projects as Anxious to Make, called "How to Make Yourself Into A Commissioning Body in 5 Easy Steps," proposes that by outsourcing all of the facets of artistic practice to gig workers and algorithms, anyone can make themselves into a creative enterprise and achieve the neoliberal dream. ${ }^{26}$ The absurdist prompt led us to develop a workshop and methodology that walks artists through common blockages and offers solutions that can be commissioned through the sharing economy, gesturing toward endless productive duplication. In this workshop we asked things like, "Do you see yourself as both an artist and 'brand' that distributes your work?" and "Do you have a growing sense of distrust in the systems that govern your life, career, community, nation, and/or planet?" We offered solutions such as "Commission a performance to externalize distrust," or a sharing-economy sourced "Brand Generator" as tools designed to outsource the anxieties that come from the loss of control or agency we face, while constantly renegotiating our shifting identities as artists navigating an economic system that subsumes creativity for its own ends.

Not far behind this mess lies another set of questions about ethics, exploitation, and the role of the artist. These questions surround how we, as artists, might unintentionally reflect and reproduce the very system we are trying to critique if we are not careful about our outsourcing practices. In December 2018, soon after we debuted "Seeing Blocks and Crypto Bros," someone tweeted at us:

does the show address the mechanical turk labor stuff at any point? "get MT workers to complete an art prompt" is Bad". - @fascinated, Dec 16, 2018 27

A Twitter debate over our commissioned drawings ensued, with another user generously explaining our project and valuing it for its depiction of many attempts to visualize an abstract technological concept. Thereafter the same user responded:

that's pretty cool, tho this stuff still leaves me uneasy. usually there is this tongue in cheek thing with MT-made art things that suggests "we hired these random workers and look how bad/simple/primitive the stuff they drew was haha we are from the artworld" @fascinated, Dec 16, $2018^{28}$

We have to admit that @fascinated has a point. The art world is notoriously bad at valuing or respecting the world outside itself, especially when it involves the kinds of labor from 
which many artists distance themselves, and especially if they, too, perform this very labor in order to survive.

In 2016, we conducted surveys with cultural workers and cloud workers, asking questions about the sharing economy, labor, bosses, and the future of work. ${ }^{29}$ Of the people we surveyed, $34 \%$ identified as artists; of these artists, a quarter identified as workers in the sharing economy, while half identified as job insecure. In this same survey we also asked, "What is something you've done for money (legal or illegal) that you would not share on your résumé or CV?" Responses to this question included: drug dealing, sex work, taking psychological tests, participating in borderline academic fraud, and running various buy-and-return schemes at giant corporate retailers. One respondent said, "Amazon MTurk work is something I would not list [on my résumé]. I see myself doing more of this work in the near future, but less in the long term., ${ }^{30}$

In observing the mixture of unspoken code, class un/consciousness, and shame that often arises around artist identity and socioeconomic class privileges, what is clear to us now is that while the precarious conditions that surround artists are nothing new, the old categories for describing different types of laborers or their respective classes no longer hold.

Moreover, after three years of outsourcing our work, what is even clearer is that the internet savvy MTurker or sharing economy worker with multiple side-gigs is keenly aware of the economic destabilization that automation and outsourcing are already bringing, and will continue to bring in the coming years. As one of the respondents explains in "The Future of Work: Testimonials," our video compilation of interviews with cloud workers:

The way I see it, we've got different tiers: we've got those at the bottom, those at the middle, and those at the upper, the rich, the $1 \% \ldots$ which the $99 \%$ of us are not. And us, being the regular workers, we kind of get in where we fit in.... So, I feel like a worker, but I don't feel like I'm really getting my fair share of things because of the way the economy is skewed. ${ }^{31}$

When asked about automation and the future of work, this respondent continues:

A ton of people are going to lose jobs and machines are going to take the place of those jobs that we provide our families with. Fast food workers are going to be replaced with technology. Self-driving cars are coming, so there aren't gonna [sic] be any more taxi jobs. Janitorial jobs, maintenance jobs.... The rich, the 1\% I was talking about are gonna [sic] have technology replace those jobs. And a lot of the people who work those jobs are gonna [sic] be out of work. ${ }^{32}$ 
These workers are also the ones organizing and creating online spaces that offer resources, tools, and support to cloud workers. In "A Crowded Future: Working against Abstraction on Turker Nation," Kathryn Zyskowski and Kristy Milland show how workers on Turker Nation, a workerrun online community of MTurkers, ${ }^{33}$ use the platform to structure time, build socializing spaces at work, and initiate collective organizing:

In an ideal world, what does a union do? Provide support for workers, stand up for workers rights and values, and support better working conditions. We have that all here at Turker Nation. ${ }^{34}$

Using feminist methodologies, Zyskowski and Milland reject the terms "immaterial labor" and "affective labor" used by Italian school theorists Maurizio Lazzarato, Michael Hardt, and Franco Berardi to describe the work of MTurkers. Instead they choose Nancy Baym's term "relational labor" as the best descriptor. Baym, who did research on how musicians connect with audiences through digital platforms, defines relational labor as the "regular, ongoing communication with audiences over time to build social relationships that foster paid work." ${ }^{35}$ Zyskowski and Milland explain that while the labor of relationship building is unpaid, it is understood as one of the most effective ways to promote the music, sell merchandise, and drive ticket sales. They conclude, "While artists are being told to 'connect' with audiences over social media, Turkers have built supporting sites (such as Turker Nation) precisely because there was no way to connect with other workers or Requesters. ${ }^{.36}$

When it comes to creating publics, mobilizing, and taking collective action, MTurkers have achieved some minor victories. Most of these victories have taken the form of small or short-term collective actions. ${ }^{37}$ For instance, in July of 2014, a spammy HIT was posted to Mechanical Turk by an academic researcher. The HIT - though far from the first example of academic exploitation on the platform-led angry MTurkers to create a set of ethical guidelines for university institutional review boards (IRBs) to use for approving academic research on Mechanical Turk. This protocol was developed alongside a group of academics at UC San Diego and Stanford, who created Dynamo, a platform to support the MTurk community in facilitating collective action. ${ }^{38}$ Another collective action facilitated by Dynamo was a letter-writing campaign to Jeff Bezos, which aimed to humanize MTurkers, who were often portrayed in the media as "downtrodden, disempowered cogs in a machine." ${ }^{39}$ The letters, which MTurkers chose to share publicly, described their work, their personal lives, and the challenges they faced on Amazon's platform. The campaign was successful in receiving positive, international press coverage for MTurk workers.

The academics working on Dynamo noted that such publicity stunts are a common tactic for labor organizers to garner recognition and begin to change labor injustices. However, such recognition is even harder to achieve for online workers, who are invisible laborers cloistered behind their computers, since "gaining visibility and recognition as 'human beings with rights' is a task in 
itself." ${ }^{40}$ Furthermore, they note that the challenges of online coordination, divided loyalties, and the risks that agitation may expose participants to harm make it difficult to sustain or scale efforts. $^{41}$

Like MTurkers, artists have tried to engage in labor organizing in the past. Take the labor concept of a strike: in 1979, conceptual artist Goran Djordjevic called for a general art strike to protest artists' alienation from the results of their labor. Artists replied to Djordjevic's call with doubts that a strike could work, ${ }^{42}$ or even stated that they were already on strike (if they were not producing new work, for instance). ${ }^{43}$ Djordjevic's strike confounded ideas of how a strike generally works: a strike removes needed labor from an employer who must make concessions to restore it. But today, "no one working in the art field expects his or her labor to be irreplaceable or even mildly important anymore," writes Hito Steyerl. ${ }^{44}$ Or as art historian Julia Byran-Wilson put it, "What does it mean for artists to go on strike when there is no consolidated workplace? If art is, by nature, individual, how do artists organize for a broadly based mass action?"45 Djordjevic's strike was ultimately cancelled for lack of participation. Yet workers in the gig economy, who likewise lack a consolidated workplace, have managed to strike against their precarious and low wage work. ${ }^{46}$

Our work with Mechanical Turkers reminds us that art cannot escape the cycles of production, consumption, and subjugation to financial domination - a domination that is made all the more evident by accelerated capitalism and its push towards the globalized decentralization of cryptocurrency. What will the next transformation of the economy look like? We're not sure, but we hope that when it occurs, artists will use its mechanisms to critique it ae well — and like its workers, learn how to organize, leverage the power of collectives, strengthen relationships of solidarity among different types of workers, cultivate more honesty and transparency around class privilege, and, of course, strike.

\section{ACKNOWLEDGEMENTS}

This project was produced for Eternal Boy Playound (2018), Anxious to Make's solo show at Telematic (San Francisco, CA). The artists thank Telematic for its support, as well as the Foundation for Contemporary Art (New York, NY) and Squeaky Wheel Film \& Media Art Center (Buffalo, NY). We thank the MTurkers who drew these brilliant drawings, and volunteerrun platforms like MTurk Crowd, Turker View, and Turker Nation that have worked to form publics and mobilize MTurkers even while a union for the platform's workers remains currently out of reach.

\section{ENDNOTES}

1. Casilli, Antonio A. "Global Digital Culture | Digital Labor Studies Go Global: Toward a Digital Decolonial Turn.” International Journal of Communication 11 (September 29, 2017), 3934-3954. https://ijoc.org/index.php/ijoc/article/view/6349. 
2. Berdugo, Liat, and Emily Martinez. "Quitting.” Anxious to Make. Accessed July 30, 2019. http://www.anxioustomake.ga/quitting.html.

3. Reese, Hope, and Nick Heath. "Inside Amazon's Clickworker Platform: How Half a Million People Are Being Paid Pennies to Train AI.” TechRepublic, December 16, 2016.

https://www.techrepublic.com/article/inside-amazons-clickworker-platform-how-half-a-millionpeople-are-training-ai-for-pennies-per-task/.

4. Hall, Miranda. "The Ghost of the Mechanical Turk." Jacobin, December 16, 2017.

https://jacobinmag.com/2017/12/middle-east-digital-labor-microwork-gaza-refugees-amazon.

5. Bowles, Nellie. "Making a Crypto Utopia in Puerto Rico." The New York Times, February 2, 2018. https:/www.nytimes.com/2018/02/02/technology/cryptocurrency-puerto-rico.html.

6. Nakamoto, Satoshi. "Bitcoin Open Source Implementation of P2P currency." P2P Foundation, February 11, 2009. http://p2pfoundation.ning.com/forum/topics/bitcoin-open-source.

7. "FAQs" Amazon Mechanical Turk. Accessed October 18, 2019. https://www.mturk.com/help. 8. "Masters Qualification Info - Everything You Need To Know.” MTurk Crowd, July 4, 2016. https://www.mturkcrowd .com/threads/masters-qualification-info-everything-you-need-toknow.1453/.

9. "If You Need More Proof That Amazon Master Qualification Is Useless and Arbitrary...." Tips for Requesters On Mechanical Turk, May 7, 2015. http://turkrequesters.blogspot.com/2015/05/ifyou-needed-more-proof-that-amazon.html.

10. “Amazon Mechanical Turk Getting Started Guide,” 2017. Accessed July 30, 2019.

https://docs.aws.amazon.com/AWSMechTurk/latest/AWSMechanicalTurkGettingStartedGuide/a mt-gsg.pdf\#Welcome.

11. Difallah, Djellel, Elena Filatova, and Panos Ipeirotis. "Demographics and Dynamics of Mechanical Turk Workers." In Proceedings of the Eleventh ACM International Conference on Web Search and Data Mining - WSDM '18, 135-143. Marina Del Rey, CA, USA: ACM Press, 2018. https://doi.org/10.1145/3159652.3159661. Interestingly, the authors of this paper mobilized ecological research on water birds to calculate the Mechanical Turk workforce, leaning on a 1930 paper titled "Calculating Waterfowl Abundance on the Basis of Banding Returns" (Washington, DC: U.S. G.P.O.).

12. DeFabio, Cara Rose. "Men Explain Blockchain to Me." Medium, October 10, 2018. https://medium.com/s/story/men-explain-blockchain-to-me-6aab55c8f15f.

13. Berdugo, Liat, and Emily Martinez. Seeing Blocks and Crypto Bros, 2018.

https://anxioustomake.ga/seeing-blocks-and-crypto-bros.

14. Sheldon, Blythe. "Crypto-Bros Beware: These Artists Aren't Buying Your Version of Utopia I KQED Arts.” KQED Arts, January 28, 2019. https://www.kqed .org/arts/13849586/crypto-broseternal-boy-playground-anxious-to-make.

15. Golumbia, David. The Politics of Bitcoin: Software as Right-Wing Extremism. Forerunners: Ideas First. Minneapolis: University of Minnesota Press, 2016.

16. Koblin, Aaron. “The Sheep Market,” 2006. Accessed July 30, 2019.

http://www.thesheepmarket.com/. 
17. Valla, Clement. A Sequence of Lines Traced by Five Hundred Individuals, 2011. Accessed July 30, 2019. https://vimeo.com/18998570.

18. McCarthy, Lauren. Social Turkers, 2013. Accessed October 18, 2019. http://socialturkers.com/.

19. Sesser, Stan. "The Art Assembly Line." Wall Street Journal, June 3, 2011, sec. Life and Style. https://www.wsj.com/articles/SB10001424052702303745304576357681741418282.

20. Tarrant, Alexander. Outsource Show, 2016. Accessed October 18, 2019.

https://alexandertarrant.com/outsource-show/.

21. Mandiberg, Michael. Postmodern Times, 2018. Accessed October 22, 2019.

https://www.mandiberg.com/postmodern-times/.

22. Johnson, Ken. “Agnieszka Kurant: 'Variables'.” The New York Times, October 9, 2014.

https://www.nytimes.com/2014/10/10/arts/design/agnieszka-kurant-variables.html.

23. Bishop, Claire. "Delegated Performance: Outsourcing Authenticity." October Vol. 140 (Spring 2012): 91-112.

24. Molesworth, Helen. Work Ethic. University Park, PA: Penn State Press, 2003, 17.

25. Ibid.

26. Berdugo, Liat, and Emily Martinez. "How to Make Yourself Into A Commissioning Body in 5 Easy Steps." Anxious to Make, 2016. Accessed July 30, 2019. http://www .anxioustomake.ga/. 27. Anthony V., Twitter post, December 16, 2018, 11:33am, https://twitter.com/fascinated/status/1074387130670034949?s=20.

28. Anthony V., Twitter post, December 16, 2018, 11:41am, https://twitter.com/fascinated/status/1074389034129084417?s=20.

29. Berdugo, Liat, and Emily Martinez. The Future of Work, 2016. V2_ Institute for the Unstable Media. http://v2.nl/archive/articles/the-future-of-work.

30. Ibid., 105.

31. Berdugo, Liat, and Emily Martinez. "The Future of Work, Testimonials." Anxious to Make. Accessed Oct 19, 2019. https://anxioustomake.ga/futureofwork.html.

32. Ibid.

33. The turkernation.com website closed Oct 2018 and moved to Slack (https://turkernation.slack.com) and Reddit (www.reddit.com/r/TurkerNation/).

34. Turker Nation, quoted in Milland, Kristy, and Kathryn Zyskowski. "A Crowded Future: Working against Abstraction on Turker Nation." Catalyst Journal: feminism, theory, technoscience 2, Issue 4, Oct 16, 2018, 2.

https://catalystjournal.org/index.php/catalyst/article/view/29581.

35. Baym, Nancy K, "Connect With Your Audience! The Relational Labor of Connection." The Communication Review Journal, Volume 18, 2015 - Issue 1: The Future of Feminist Media Studies, Mar 20, 2015, 16. www.tandfonline.com/doi/abs/10.1080/10714421.2015.996401 36. Milland, Kristy, and Kathryn Zyskowski. "A Crowded Future: Working against Abstraction on Turker Nation." Catalyst Journal: feminism, theory, technoscience, 2, Issue 4, Oct 16, 2018 , 9. https://catalystjournal.org/index.php/catalyst/article/view/29581. 
37. Salehi, Niloufar, and Lilly C. Irani, Michael S. Bernstein, Ali Alkhatib, Eva Ogbe, Kristy Milland, Clickhappier. "We Are Dynamo: Overcoming Stalling and Friction in Collective Action

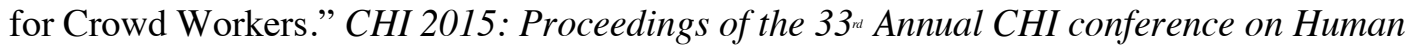
Factors in Computing Systems, 2015, 1621-1630, https:/hci.stanford.edu/publications/2015/dynamo/DynamoCHI2015.pdf.

38. Ibid. Note that Dynamo used to be located at www.wearedynamo.org, a site that is now defunct.

39. Ibid., 1626.

40. Ibid.

41. Ibid.

42. Apostol, Corina L. "Art Workers, Art Strikes and Collective Actions." ArtLeaks Gazette, September 2017.

https://artsleaks.files.wordpress.com/2012/09/09 apostol algazette4 2017 150dpi.pdf.

43. Steyerl, Hito. Duty Free Art. London and New York: Verso, 2017.

44. Ibid., 22.

45. Bryan-Wilson, Julia. "Strike." In Work Ethic, edited by Helen Molesworth. University Park, PA: Penn State Press, 2003, 214.

46. In 2016 couriers working for the German food delivery company Foodora (akin to UberEats) garnered significant media attention when they went on strike in Italy, as an instantiation of the first ever self-organized strike in the Italian gig economy. Tassinari, Arianna, and Vincenzo Maccarrone. "Striking the Startups." Jacobin, January 23, 2017.

https://jacobinmag.com/2017/01/foodora-strike-turin-gig-economy-startups-uber.

\section{AUTHOR BIOS}

Anxious to Make is the collaborative practice of Liat Berdugo and Emily Martinez, two commissioning bodies. We focus on economic concepts, such as cryptocurrencies and the socalled "sharing economy," and the accelerationist, neoliberal landscapes associated with them. Our work examines how these economic concepts intersect with colonialism, technology, wealth culture, race, altruism, utopianism, and exploitation. While Anxious to Make's physical existence takes many shifting forms, it often manifests as series of video commissions, downloads, online generators, workshops, net art interventions, books, and sweepstakes. Anxious to Make believes in absurdist extremes as way to examine contemporary realities. Our work has appeared recently in EMMEDIA (Calgary, CA), Transmediale (Berlin, DE), Yerba Buena Center for the Arts (San Francisco), MoMA PS1 (New York), V2_Lab for the Unstable Media (Rotterdam, NL), The Luminary (St. Louis), The Institute of Network Cultures (Amsterdam, NL), The Wrong Biennale, and Telematic (San Francisco). More at http://anxioustomake.ga/.

Liat Berdugo is an artist, writer, and curator whose work focuses on embodiment and digitality, archive theory, and new economies. Her work has been exhibited in galleries and festivals 
internationally, and she collaborates widely with individuals and archives. She is an assistant professor of Art + Architecture at the University of San Francisco, and is currently writing a book about the weaponization of cameras in Israel/Palestine (forthcoming, Bloomsbury). More at liatberdugo.com.

Emily Martinez is a queer Latinx, 1st generation Cuban immigrant/refugee raised in Miami and now living in Los Angeles. She is a new media artist, digital strategist, developer, educator, activist, and serial collaborator who believes in the tactical misuse of technology. Her most recent works take on the sharing economy, digital labor struggles, algorithmic bias, surveillance capitalism, crypto colonialism, tech bros, and tech culture at large. Long-term projects/departures/returns explore trauma, queerness, and in/visibility. When not making art, Emily works with Color $<$ Coded $>$, a POC tech and pedagogy collective in Los Angeles. More at somethingnothing.me. 